random effects meta-analyses, to obtain summary effect estimates for the effect of depression on hypertension, pooling hazard ratios and odds ratios separately. We also separately combined studies which defined depression as a categorical or a continuous variable.

Results After de-duplication, the search identified 7402 studies. Twenty-two studies were eligible for inclusion in the review, 17 of which were included in the meta-analyses. Meta-analyses showed an increased hypertension risk among depressed versus non-depressed participants (pooled OR: 1.31, 95\% CI: 1.051.64; pooled HR: 1.18, 95\% CI: 1.02-1.36). Among studies which assessed depressive symptoms on continuous scales meta-analyses indicated an increased risk with every unit increase on the depressive symptoms scale (pooled OR: 1.06, 95\% CI: 0.97-1.16; pooled HR: 1.06, 95\% CI: 1.01-1.12).

Discussion Our review findings provide evidence that depression may be associated with an increased risk of hypertension. However, existing studies have important limitations and the substantial heterogeneity between studies included in two of the four meta-analyses remained unexplained after performing subgroup analyses. Before concluding that depression is indeed associated with an increased risk of hypertension, future prospective studies should improve the accuracy of exposure and outcome assessment, aim to take all major confounding and effect modifying factors into account, and present effect estimates for subgroups in order to help facilitate more meaningful meta-analyses of study findings. Further research is also needed to determine whether the observed association between depression and hypertension is causal.

\section{P44 SELF-HARM, VIOLENCE AND PREMATURE DEATH AMONG YOUNG PERSONS WHO EXPERIENCED TRAUMA-RELATED HOSPITALISATION DURING CHILDHOOD: A NATIONAL REGISTER-BASED COHORT STUDY}

${ }^{1} \mathrm{RT}$ Webb*, ${ }^{2,3} \mathrm{~S}$ Antonsen, ${ }^{1} \mathrm{MJ}$ Carr, ${ }^{1} \mathrm{~L}$ Appleby, ${ }^{2,3} \mathrm{CB}$ Pedersen, ${ }^{1} \mathrm{PLH}$ Mok. ${ }^{1}$ Centre for Mental Health and Safety, The University of Manchester, Manchester UK; ${ }^{2}$ Centre for Integrated Register-based Research (CIRRAU), Aarhus University, Aarhus, Denmark; ${ }^{3}$ National Centre for Register-based Research, Aarhus University, Aarhus, Denmark

\subsection{6/jech-2017-SSMAbstracts. 146}

Background Epidemiological research has reported strong links between trauma-related hospitalisation and future risks of fatal and nonfatal adverse outcomes. However, some important research questions remain unanswered, including association with hospitalisation occurring specifically during childhood, longer-term follow-up from mid-adolescence through the earlier stages of adulthood, assessment of self-harm versus violence risks in the same study population, and absolute risk estimation. To reduce risk in young people, clinicians and public health experts require a better knowledge of subsequent pathways for individuals who experience trauma-related hospitalisation during childhood.

Methods This national cohort study examined $n=1,087,672$ persons born in Denmark 1977-1997 with complete linkage to national psychiatric, general hospital and crime registers. Survival analyses (C) SAS Institute Inc.) was used to estimate incidence rate ratios (IRRs) for self-harm, violent criminality, interpersonal violence injury, and all-cause mortality between 15 th and 35 th birthdays among cohort members with and without trauma-related hospitalisation prior to 15 th birthday. Accounting for competing risks, cumulative incidence percentage values were estimated to age 35. Estimates were stratified by gender and by reason for hospitalisation during childhood: self-harm, interpersonal violence or accident.

Results Risk for each adverse outcome assessed was raised among young persons who experienced trauma-related hospitalisation at least once during childhood. Confounding by parental socioeconomic status, measured according to income, educational attainment and employment status, explained little of these risk elevations. Individuals hospitalised during childhood following self-harm or interpersonal violence had much higher risks for self-harm and violent criminality aged 15-35 years. Some particularly high cumulative incidence values were observed: subsequent violent offending in males hospitalised following interpersonal violence during childhood, $25.0 \%$ (95\% CI 21.2-28.9); later self-harm in females hospitalised following interpersonal violence, $18.3 \%$ (95\% CI 13.5-23.6) and following self-harm during childhood, 21.4\% (95\% CI 19.8-23.1). More frequent trauma-related hospitalisations, and hospitalisations for multiple trauma types at such an early age, conferred marked risk elevations through young adulthood.

Conclusion Although not all episodes of self-harm and interpersonal violence in the community are routinely captured via hospital records, trauma-related hospitalisation during upbringing may be a clinically useful marker for familial dysfunction and childhood distress that subsequently predicts internalised and externalised destructive behaviours among youths and young adults. Comprehensive national guidelines are needed to tackle the multifaceted vulnerabilities of children hospitalised for injuries or poisonings.Healthcare, social services and educational workers must provide particularly robust support to children hospitalised following self-harm or interpersonal violence, and those who experience multiple trauma-related hospitalisations during upbringing.

\section{P45 SOCIAL AND SPATIAL MOBILITY AND SELF-REPORTED HEALTH IN OLDER-AGE: LINKAGE OF THE SCOTTISH LONGITUDINAL STUDY TO THE 1947 SCOTTISH MENTAL SURVEY}

\begin{abstract}
${ }^{1,2}$ LF Forrest ${ }^{*},{ }^{1,2} \mathrm{C}$ Dibben, ${ }^{1,2} \mathrm{Z}$ Feng, ${ }^{3}$ I Deary, ${ }^{4} \mathrm{~F}$ Popham. ${ }^{1}$ Administrative Data Research Centre Scotland, University of Edinburgh, Edinburgh, UK; ${ }^{2}$ School of Geosciences, University of Edinburgh, Edinburgh, UK; ${ }^{3}$ Centre for Cognitive Ageing and Cognitive Epidemiology, University of Edinburgh, Edinburgh, UK; ${ }^{4} \mathrm{MRC/CSO}$ Social and Public Health Sciences Unit, University of Glasgow, Glasgow, UK
\end{abstract}

\subsection{6/jech-2017-SSMAbstracts. 147}

Background The use of administrative datasets to create new cohorts with large sample sizes allows us to answer research questions that we previously could not.Linkage to historic datasets allows exploration of factors that may be important across the life course.

There is debate within the literature as to whether social mobility inflates or constrains health inequalities. The role of geographical mobility is unknown. We were interested in exploring how spatial and social mobility might impact on health in older age using linked administrative and cohort data.

Methods The 1947 Scottish Mental Survey (a 1936 birth cohort of 70805 individuals with age 11 cognitive ability test scores) was linked to the Scottish Longitudinal Study (a semirandom sample of $5.3 \%$ of the Scottish population), and backward linked to the 1939 register to obtain parental occupation in 1939 (as a measure of social origin) and forward linked to 
obtain occupation from the 1991 census (social destination), as well as geographical location in 1939 and 1991. We examined the movement between three geographical areas (Edinburgh, Glasgow, Other) in Scotland. Four social mobility trajectories were derived. We modelled the relationship between social and geographic mobility and likelihood of having self-reported limiting long term illness (LLTI) at age 65, using logistic regression.

Results Those who were geographically mobile to Edinburgh had the lowest rates of self-reported LLTI and those who remained resident in the Glasgow area had the highest rates. The lowest and highest rates of LLTI were found in the socially-static at the top and bottom of the social scale respectively, with intermediate rates seen in the upwardly and downwardly mobile. However neither social nor spatial mobility were significantly associated with health in later life in the fully adjusted model when highest educational qualifications and cognitive ability were included. Being female, having higher education qualifications and being in a higher social class in childhood and adulthood reduced the likelihood of poor health at age 65 .

Conclusion Although both social class and geographical location were associated with the likelihood of LLTI in later life, social and spatial mobility were not, when factors such as education and cognitive ability were controlled for.

\section{P47 ARE OBESITY AND INFLAMMATION FROM MIDLIFE ASSOCIATED WITH PHYSICAL FATIGABILITY IN OLD AGE? FINDINGS FROM A BRITISH BIRTH COHORT STUDY}

${ }^{1} \mathrm{R}$ Cooper*, ${ }^{1} \mathrm{M}$ Popham, ${ }^{2} \mathrm{AJ}$ Santanasto, ${ }^{2} \mathrm{NW}$ Glynn, ${ }^{1} \mathrm{D}$ Kuh. ${ }^{1} \mathrm{MRC}$ Unit for Lifelong Health and Ageing, UCL, London, UK; ${ }^{2}$ Department of Epidemiology, University of Pittsburgh, Pittsburgh, USA

\subsection{6/jech-2017-SSMAbstracts. 148}

Background Physical fatigability, the level of fatigue experienced while undertaking specified physical tasks, increases with age throughout adulthood. These age-related changes, which reflect reductions in energy availability, precipitate declines in activity participation and function. Higher levels of fatigue are also related to increased risk of disability and premature mortality so it is imperative to identify modifiable risk factors across life associated with physical fatigability. Crosssectional analyses suggest that obesity and inflammation may be associated with increased risk of high physical fatigability. However, whether these inter-related factors act on the same pathway is unclear and requires further investigation in longitudinal studies. We thus aimed to examine the associations of body mass index (BMI) and inflammatory markers from midlife with subsequent levels of perceived physical fatigability in a nationally representative sample.

Methods Up to 2095 men and women from the MRC National Survey of Health and Development, a British cohort followed-up prospectively since birth in 1946, who had valid physical fatigability scores on the Pittsburgh Fatigability Scale (PFS) at age 68 years were included in analyses. Linear regression models were used to assess associations of BMI from age 43 and inflammatory markers (C-reactive protein (CRP) and interleukin-6 (IL-6)) at age 60-64 with continuous PFS scores at age 68. Adjustments were made for sex (where no evidence of interaction was found), long-term limiting illness, physical activity and symptoms of anxiety and depression. All analyses were performed using STATA v14.2.

Results Women had higher mean PFS scores than men (16.0 $(\mathrm{SD}=9.3)$ vs $13.4(\mathrm{SD}=9.0), \mathrm{p}<0.01)$, with higher scores indicating greater perceived physical fatigability. In sex-adjusted analyses, higher BMI and higher levels of CRP and IL-6 were associated with higher PFS scores. For example, participants with $\mathrm{BMI} \geq 30 \mathrm{~kg} / \mathrm{m}^{2}$ at age 43 had sex-adjusted mean PFS scores 4.7 (95\% CI: 3.3-6.1) points higher than those with BMI $20-24.9 \mathrm{~kg} / \mathrm{m}^{2}$ and, those in the highest fifth of IL-6 at age 60-64 had a mean score 4.9 (95\% CI: 3.5-6.3) points higher than those in the bottom fifth. When these associations were mutually adjusted and adjusted for other covariates, higher BMI and IL-6 remained associated with higher PFS scores, whereas associations with CRP were fully attenuated.

Conclusion These findings highlight the potentially important influence of inflammatory and other cardio-metabolic processes on physical fatigability. They suggest that both BMI and inflammation from midlife may be important targets for intervention to reduce the burden of this commonly reported symptom in older populations.

\section{P48 COMPARING BMI WITH SKINFOLDS TO ESTIMATE AGE AT ADIPOSITY REBOUND AND ITS ASSOCIATIONS WITH LATER CARDIO-METABOLIC RISK MARKERS}

${ }^{1} \mathrm{C}$ Di Gravio*, ${ }^{2} \mathrm{GV}$ Krishnaveni, ${ }^{2} \mathrm{R}$ Somashekara, ${ }^{2} \mathrm{SR}$ Veena, ${ }^{2} \mathrm{~K}$ Kumaran, ${ }^{2} \mathrm{M}$ Krishna, ${ }^{2} \mathrm{SC}$ Karat, ${ }^{1} \mathrm{CHD}$ Fall. ${ }^{1} \mathrm{MRC}$ Lifecourse Epidemiology Unit, University of Southampton, Southampton, UK; ${ }^{2}$ Epidemiology Research Unit, CSI Holdsworth Memorial Hospital, Mysore, India

\subsection{6/jech-2017-SSMAbstracts. 149}

Background Adiposity rebound (AR), defined as the lowest point before the second rise in body mass index (BMI), occurs between the ages of 5 and 7. Early age at AR is associated with higher risk of obesity in later life. However, BMI as a measure of adiposity has limitations: first, BMI incorporates both fat and lean mass, second, BMI is calculated from both height and weight. To identify the AR, a direct measure of fat (i.e. skinfold thickness) might be more relevant. We used data from the Mysore Parthenon Birth Cohort to compare relative merits of BMI and skinfolds in identifying $\mathrm{AR}$ and predicting BMI and cardio-metabolic risk factors at 13.5 years.

Methods The cohort was set up in 1997 in Mysore, India, to examine the long-term effects of gestational diabetes on cardiovascular disease risk factors in the offspring. Children were followed-up annually until 5 years, and 6-monthly after that for detailed anthropometry. At 13.5 years, 545 children had measurements of cardio-metabolic risk markers. We used nonlinear splines and regression analyses (STATA version 14) to characterise the subject-specific growth of BMI and skinfolds (sum of triceps and subscapular skinfolds) throughout childhood, and to assess the associations between age at AR, BMI and cardio-metabolic risk factors.

Results BMI and skinfolds had similar trajectories with both reaching their minimum between 5 and 6 years. Average age at AR was similar between the two measures (5.94 years and 5.73 years respectively), with skinfold-derived AR being characterised by higher variability (standard deviation: 1.47 years and 2.18 years respectively). Later age of BMI-derived AR was associated with lower BMI $\left(-0.89 \mathrm{~kg} / \mathrm{m}^{2} ; 95 \% \mathrm{CI}\right.$ : $\left.\left[-1.04,-0.74 \mathrm{~kg} / \mathrm{m}^{2}\right]\right)$, fat mass $(-1.14 \mathrm{~kg} ; 95 \% \mathrm{CI}:[-1.36,-$ $0.91 \mathrm{~kg}])$, HOMA-IR $(-0.12 ; 95 \% \mathrm{CI}:[-0.17,-0.07 \mathrm{~kg}])$ and 